\title{
Benefícios do desenvolvimento de projetos de inovação tecnológica
}

\author{
Aline Bellintani Caluigaris, Msc. \\ Universidade Federal de São Carlos - DEP/UFSCar \\ E-mail:aline@dep.ufscar.br \\ Ana lúcia Vitale Torkomian, Dra. \\ Universidade Federal São Carlos - DEP/UFSCar \\ E-mail: torkomia@power.ufscar.br
}

\section{Resumo}

O objetivo deste artigo é apresentar os benefícios obtidos com a realização de projetos de inovação tecnológica, por meio de três programas governamentais específicos. Os dados apresentados são resultados de uma pesquisa qualitativa em que foi realizado um estudo de caso, com interesse em caracterizar a cooperação entre a Universidade Federal de São Carlos (UFSCar) e o setor empresarial, que ocorre com a utilização de programas de inovação tecnológica específicos. A partir desse estudo pôde-se verificar que os principais benefícios são, de acordo com os discursos dos envolvidos na execução dos projetos, o desenvolvimento das próprias inovações desenvolvidas e os resultados práticos obtidos tanto para o meio acadêmico como para o empresarial.

\section{Palavras-chave}

Benefícios, inovações tecnológicas, cooperação universidade-empresa.

\section{Benefits from development of technological innovation projects}

\begin{abstract}
The objective of this article is to present the benefits obtained with the technological innovation programs by government. This paper presents results of a qualitative research related to a case study characterizing the Federal University of Sao Carlos city-industry cooperation using technological innovation programs. Several researches, entrepreneurs and consultants were interviewed. The speech analysis for these interviews shows that main benefits and developed innovations and practical results for both academic and industrial sectors.
\end{abstract}

\section{Key words}

Benefits, technological innovation, university-industry cooperation. 


\section{INTRODUC̣̃̃O}

Com a finalidade de aumentar a capacitação tecnológica, visando adaptarem-se às demandas do mercado, empresas nacionais têm buscado vantagens competitivas por meio da introdução de inovações tecnológicas. Essa tarefa, entretanto, é dificultada pelo reduzido número de pesquisadores nas empresas brasileiras.

Buscando superar este obstáculo, o governo (federal e estadual) tem criado programas de financiamento que favorecem a cooperação entre universidade (fonte de pesquisadores) e empresas no desenvolvimento de inovações. Mesmo com os recursos governamentais disponibilizados, a demanda por tais projetos, no entanto, tem sido considerada pequena. Considerando-se estes aspectos, percebeu-se a importância de uma análise da relação universidade-empresa, buscando investigar o funcionamento, o tipo de relacionamento e a eficiência dos programas governamentais de financiamento para projetos que visam a cooperação.

Nesse contexto, foi estudada a cooperação entre a Universidade Federal de São Carlos (UFSCar) e o setor empresarial, por meio de determinados programas governamentais de inovação tecnológica, visando a identificação de características específicas desse relacionamento (CALLIGARIS, 2002). Assim, foram identificadas variáveis associadas aos benefícios, dificuldades e motivações associadas ao desenvolvimento dos projetos.

Neste artigo são apresentados os benefícios obtidos em decorrência do desenvolvimento de projetos de inovação. $\mathrm{O}$ objetivo é analisar a forma como esses resultados poderiam auxiliar a otimização de projetos de inovação, atingindo tanto os interesses das empresas como os das universidades.

Inicialmente será apresentada uma breve revisão teórica do tema abordado, seguida da apresentação de alguns aspectos do caso da UFSCar, para balizar os resultados apresentados posteriormente.

\section{REVISÃO DE LITERATURA}

Ao longo dos anos, os sistemas produtivos têm modificado suas estruturas organizacionais de acordo com o mercado e o ambiente em que estão inseridos. Nesse processo de mudança, novas tecnologias de produto e processo produtivo estão sendo introduzidas nas empresas (PEDRASSOLI NETO et al., 2001), o que tem possibilitado o desenvolvimento de inovações tecnológicas.

A realização de inovações tecnológicas ocorre em função dos aspectos internos e externos às empresas. Os primeiros referem-se à forma organizacional, envolvendo planejamento estratégico, aprendizagem e competência de toda a empresa. Os outros estão vinculados ao relacionamento com o mercado e o ambiente que as circunda, abran- gendo os consumidores, os fornecedores e os concorrentes, entre outros (SILVA, 1999).

As inovações, segundo Shumpeter (1982), assim como a tecnologia, estão no centro do desenvolvimento tecnológico e devem viabilizar-se à medida que atendam às necessidades sociais e de mercado. Nesse sentido, de acordo com esse autor, o desenvolvimento econômico só ocorrerá se houver uma demanda por novos produtos e métodos produtivos.

Este desenvolvimento efetiva-se a partir de inovação, ou seja, de novas combinações de materiais e forças diferentes, empregadas de forma diversa - como por exemplo a introdução de um novo bem de produção ou uma nova qualidade atribuída a um bem preexistente (SHUMPETER, 1982). Em um sentido mais amplo, o termo inovação inclui processos por intermédio dos quais as empresas desenvolvem, coordenam e levam à prática seus novos projetos de produtos e processos de produção (OLIVEIRA, 2001).

As inovações tecnológicas realizadas nas empresas podem ser divididas em: substituição de equipamentos e/ou aquisição de novos conhecimentos e métodos, como menciona Porter (1993).

Para realizarem inovações, ou seja, viabilizar novos produtos, melhorar a qualidade dos existentes e diminuir os custos de produção, atividades de Pesquisa e Desenvolvimento (P\&D) em uma empresa são vistas como fundamentais. A atividade de Pesquisa envolve a geração de novos conhecimentos e a de Desenvolvimento visa à aplicação dos conhecimentos gerados, objetivando a criação de novos produtos ou o aperfeiçoamento dos existentes, com a intenção de torná-los melhores e mais baratos (FERRO, 1997).

No Brasil os investimentos das indústrias em P\&D são muito pequenos e os recursos governamentais ainda são a principal fonte para o desenvolvimento de pesquisa no País (SILVA, 1999). Uma das razões é que, nos países em desenvolvimento, o senso comum remete à idéia de que a pesquisa é uma atividade da universidade. Esse fato pode ser observado no Brasil (CRUZ, 2000), onde quase a totalidade da pesquisa é realizada pelo setor acadêmico.

A realização de pesquisa é uma das funções da universidade, mas isso não implica, em nenhuma instância, que as atividades de P\&D devam ser realizadas exclusivamente por essas instituições, e não pelas empresas. Cruz (1998) e Nelson \& Rosemberg (1993) mencionam que deveria haver no Brasil uma cultura que valorizasse a realização das atividades de P\&D nas empresas (SUTZ, 2000).

De qualquer maneira, a aproximação com a universidade facilita a realização de $\mathrm{P} \& \mathrm{D}$ nas empresas. Com a transferência de tecnologia gerada nessas instituições para o setor privado, várias dificuldades de desenvolvimento de $\mathrm{P} \& \mathrm{D}$ em micro e pequenas empresas poderiam ser solucionados (CALLIGARIS, 2002). 
A transferência de conhecimento da universidade para a empresa e vice-versa é uma maneira de ambas cooperarem, beneficiando-se mutuamente. A cooperação universidadeempresa tem incentivado uma melhora na qualidade do ensino universitário, a atuação do meio acadêmico na sociedade (SEGATTO \& SBRAGIA, 1996) e a obtenção de conhecimentos que podem ser utilizados no próprio segmento universitário.

A fim de incentivar o desenvolvimento de inovações tecnológicas em micro e pequenas empresas, por meio da cooperação com universidades, o governo brasileiro, nos âmbitos federal e estadual, tem proporcionado financiamento por meio de determinados programas, especialmente a partir da década de 1990.

Para descrever as relações entre universidade, empresa e governo, modelos geométricos têm sido criados ao longo dos anos. Entre eles estão: o modelo linear, o do triângulo de Sábato e o da Triple Helix (Hélice Tripla) (OLIVEIRA, 2001).

O modelo linear representa a transferência unidirecional de conhecimento do setor acadêmico para o mercado. Fundamenta-se na concepção de que o processo de inovação vincula-se às mudanças tecnológicas e é gerado pela pesquisa científica. Um modelo que foi considerado, ao longo do tempo, insuficiente por não ser capaz de refletir, segundo Schetze (1996) apud Silva (1999), a natureza multidirecional da inovação e não indicar como essa se originou.

Posteriormente, novos modelos baseados no conhecimento e voltados para o desenvolvimento econômico foram elaborados; com a inserção de um outro ator nesse cenário, o governo, um conceito multidirecional foi introduzido (OLIVEIRA, 2001). Esses modelos chamados de não-lineares, são capazes de representar as relações que ocorrem entre os setores acadêmico, produtivo e governamental, refletindo melhor a complexidade dessas relações (CALLIGARIS, 2002).

A ligação entre esses atores foi discutida primeiramente em 1968, quando Jorge Sábato e Natalino Botana propuseram um modelo representado graficamente por um triângulo, cujos vértices eram ocupados por três agentes (governo, universidade e estrutura produtiva). As inter-relações entre os setores produtivo e acadêmico são consideradas as mais importantes das três e as mais difíceis de ocorrer (PLONSKI, 1998).

Este modelo mostra a ação múltipla e coordenada entre governo, estrutura produtiva e infra-estrutura científicotecnológica. Esses três elementos são considerados como fundamentais para o desenvolvimento das sociedades contemporâneas (PLONSKI, 1998). O modelo do Triângulo de Sábato, de acordo com Brisolla (1998), propunha ações diretas do Estado para romper o isolacionismo do sistema de Ciência e Tecnologia (C\&T) em relação à base econômica. Nos países em desenvolvimento, os vértices da base deste triângulo (universidade-empresa) tendem a se constituir, conforme afirma essa autora, em pontos isolados e sem conexão.

Um modelo que permite compreender melhor as relações que se estabelecem nas três esferas institucionais - universidade, empresa e governo - foi estabelecido no final da década de 1980 e início dos anos 1990. Os autores Etzkowitz \& Peters (1991) propuseram um modelo que possui uma representação na forma de espiral, mostrando a ligação das três esferas nos vários estágios do processo de inovação. Nesse modelo, denominado Triple Helix, as partes envolvidas se relacionam simultaneamente.

A Triple Helix é um modelo alternativo para explicar o atual sistema de pesquisa no contexto social, no qual a universidade pode exercer um importante papel na inovação. É visto como um modelo analítico que agrega uma variedade de arranjos institucionais e modelos políticos para explicar suas dinâmicas (ETZKOWITZ \& LEYDERSDORFF, 2000).

Há três variações de arranjos institucionais envolvendo as relações universidade-empresa-governo, abrangendo a Triple Helix. Essas variações do modelo refletem a evolução dos sistemas de inovação e o atual conflito a respeito do caminho a ser seguido pelos atores envolvidos no processo.

A Triple Helix I é uma configuração em que o Estado abarca as relações entre a academia e a indústria. É um modelo estático das relações universidade-empresa-governo que ocorre em uma situação histórica específica como, por exemplo, na antiga União Soviética e em países do Leste Europeu. Nesse arranjo, como afirmam Etzkowitz \& Leydersdorff (2000), há apenas um pequeno espaço para iniciativas entre universidades e empresas, pois o Estado sobrepõe-se aos outros dois atores. Com este modelo a inovação é disseminada ao mesmo tempo em que é desencorajada.

Um segundo arranjo, o da Triple Helix II, é um modelo político, com esferas institucionais separadas com fortes limites dividindo-as e com relações altamente circunscritas entre essas esferas, tendo cada uma delas o seu papel totalmente definido.

Já no arranjo da Triple Helix III não existem limites entre as esferas institucionais como há no da Triple Helix II e os acordos entre os agentes são encorajados, mas não controlados pelo governo como no arranjo da Triple Helix I.

Atualmente, há uma tendência de muitos países a tentar atingir o terceiro arranjo, devido ao objetivo da realização de um ambiente inovativo, interagindo firmas geradas por spin-offs, iniciativas tri-laterais de desenvolvimento econômico e laboratórios governamentais de grupos de pesquisa 
(ETZKOWITZ \& LEYDESDORFF, 2000).

$\mathrm{Na}$ Triple Helix III há ligações entre as partes que emergem de vários estágios do processo de inovação (ETZKOWITZ \& LEYDERSDORFF, 2000). Dessa forma, ocorre uma relação dinâmica entre universidade, empresa e governo.

As políticas da Triple Helix III devem operar considerando que os aspectos envolvidos no relacionamento universidade-empresa-governo variam de um local para outro (SUTZ, 2000), dependendo de aspectos sociais, comportamentais, contextuais e estruturais diferentes.

No Brasil, há algum tempo, e mais intensamente a partir da década de 1990, agências governamentais têm demonstrado interesse em incentivar o desenvolvimento científico e tecnológico do País. Esse desenvolvimento tem sido fomentado por meio da criação de programas voltados especificamente ao desenvolvimento de inovação tecnológica nas empresas nacionais em conjunto com universidades (modelo Triple Helix III).

Com o interesse em investigar o funcionamento, o tipo de relacionamento e a eficiência dos programas governamentais de financiamento para projetos que visam a cooperação, foi realizado um estudo de caso específico envolvendo a Universidade Federal de São Carlos (UFSCar) (CALLIGARIS, 2002). As características dos programas de inovação tecnológica envolvidos neste estudo são apresentadas na sequiência.

\section{OS PROGRAMAS DE INOVAC̣ÃO TECNOLÓGICA PATME, PITE E PIPE}

Nesta seção são apresentadas características dos programas de inovação tecnológica PATME, PITE e PIPE.

\section{Programa de Apoio Tecnológico às Micro e Pequenas Empresas - PATME}

O PATME foi criado pelo SEBRAE juntamente com a FINEP para permitir que as empresas tenham acesso aos conhecimentos existentes no País, por meio de consultorias, visando à elevação de seu patamar tecnológico. Seu principal objetivo é a promoção e a otimização de processos e produtos de micro e pequenas empresas, a partir de serviços prestados por instituições tecnológicas.

Este programa possui três tipos de projetos que podem ser desenvolvidos:

- Projetos do tipo "A": que busquem resolver problemas de um determinado produto e/ou processo produtivo, com a intenção de melhorar a produtividade da empresa e diminuir os custos. Neste tipo de projeto encaixam-se ações voltadas às áreas: agroindustrial, metrologia industrial e normatização técnica;
- Projetos do tipo "B": que visem o desenvolvimento de novas tecnologias de produtos e de processos produtivos na empresa. Nesses projetos encaixam-se ações ligadas ao desenvolvimento de novos materiais, ao desenho industrial, à engenharia de processo e produto, além do desenvolvimento de software focado na produção;

- Projetos do tipo "C": de inovação tecnológica, envolvendo conhecimentos técnicos e científicos, tendo como objetivo o desenvolvimento de um produto inovador para o qual existe interesse no mercado.

O PATME financia até $70 \%$ do valor total dos projetos do tipo "A", 50\% para os do tipo "B" e $30 \%$ para os do tipo "C". Cabe às empresas envolvidas dar suas respectivas contrapartidas, dependendo do tipo de projeto em que estiverem envolvidas.

\section{Projeto de Parceria para a Inovação Tecnológica - PITE}

O PITE foi criado pela Fundação de Amparo à Pesquisa do Estado de São Paulo (FAPESP) em 1994, tendo como objetivo financiar o desenvolvimento de projetos conjuntos de inovação tecnológica entre instituições de pesquisa instaladas no Estado de São Paulo e o setor produtivo.

Neste programa a FAPESP financia, a fundo perdido, a parte do projeto sob responsabilidade da instituição de pesquisa e a empresa parceira dá uma contrapartida financeira com seus próprios recursos ou de terceiros.

Os projetos desenvolvidos por pesquisadores devem estar vinculados a empresas em áreas que envolvam problemas considerados importantes em ciência, engenharia ou em educação científica ou tecnológica.

No PITE são consideradas três modalidades de parceria envolvendo projeto conjunto de empresa ou de um grupo de empresas, que pode ser proposto por um pesquisador ou um grupo de pesquisa, ligado à universidade e/ou a outras instituições de pesquisa.

- Modalidade 1: projeto visando o desenvolvimento de inovação cuja fase exploratória esteja praticamente completada pelo pesquisador ou pelo grupo de pesquisadores que o estiverem propondo. Neste caso, a FAPESP financia até $20 \%$ do custo total do projeto;

- Modalidade 2: projeto com o objetivo de desenvolver inovação incremental, forçada pelo mercado, envolvendo normalmente as etapas de exploração e de certificação, associada a baixos riscos tecnológicos e de comercialização. Nesta modalidade, a FAPESP financia até $50 \%$ do custo total do projeto;

- Modalidade 3: projeto visando o desenvolvimento de inovação associada a altos riscos tecnológicos e baixos riscos de comercialização, mas com alto poder de "ferti- 
lizante germinativo". Nesta modalidade a FAPESP financia até $70 \%$ do custo total do projeto.

\section{Programa de Inovação Tecnológica em Pequenas Empresas - PIPE}

O PIPE é um outro programa da FAPESP, iniciado em 1997 e que tem como objetivo proporcionar apoio às pesquisas inovadoras, que possam ser executadas por pequenas empresas, localizadas no Estado de São Paulo e que possuam um alto retorno nas áreas comercial ou social.

Os projetos devem ser desenvolvidos nas empresas proponentes dos mesmos, devendo haver pesquisadores responsáveis por eles, vinculados a estas empresas por no mínimo 20 horas semanais.

O desenvolvimento de projetos PIPE compreende três fases distintas:

- Fase 1: a duração desta fase é de 6 meses e o objetivo neste período é a realização de uma pesquisa sobre a viabilidade técnica das idéias propostas. Pelo menos $2 / 3$ das atividades desta fase devem ser desenvolvidas pela pequena empresa proponente, que poderá, assim, subcontratar até $1 / 3$ de trabalhos de outras empresas, consultores ou instituição de pesquisa. Cada projeto recebe no máximo $\mathrm{R} \$ 75.000,00$ da FAPESP;

- Fase 2: essa fase tem a duração de 24 meses e nela é desenvolvida a parte principal da pesquisa. As empresas devem apresentar um plano de negócios para a comercialização, sumarizando o progresso realizado no período, descrevendo as metas para o próximo semestre. O valor máximo financiável nesta fase é de $\mathrm{R} \$ 300.000,00$ para cada projeto, sendo as concessões feitas aos projetos de maior sucesso na fase anterior. A previsão é que cerca de $1 /$ 3 dos projetos apoiados na fase 1 receberão apoio na fase 2 ;

- Fase 3: o objetivo nesta fase é o desenvolvimento de novos produtos comerciais baseados nos resultados obtidos nas fases anteriores. A FAPESP não dá apoio financeiro de qualquer natureza nesta fase, mas poderá colaborar na obtenção de apoio de outras fontes caso os resultados da pesquisa comprovem a viabilidade das idéias, bem como o seu potencial de retorno comercial.

Na seção a seguir são apresentados resultados de uma pesquisa qualitativa envolvendo a cooperação UFSCarempresa por meio dos programas de inovação tecnológica PATME, PITE e PIPE.

\section{O CASO DA UFSCar}

Os dados apresentados neste artigo consistem em resultados da pesquisa realizada por Calligaris (2002). Uma pesquisa qualitativa, de caráter exploratório, que teve como objetivo caracterizar a cooperação UFSCar-empresa por meio dos programas de inovação tecnológica PATME, PITE e PIPE.

A escolha do caso da UFSCar deve-se, entre outros motivos, ao importante papel que essa universidade tem desempenhado no contexto regional, estando inserida no Pólo de Alta Tecnologia de São Carlos - SP. Além disso, essa instituição é uma universidade de excelência que forma profissionais em diversas áreas do conhecimento; realiza projetos conjuntos com o setor empresarial gerenciados pela Fundação de Apoio Institucional ao Desenvolvimento Científico e Tecnológico (FAI/UFSCar); além de possuir um corpo docente qualificado para executar projetos de inovação tecnológica.

\section{Metodologia}

A pesquisa realizada teve como objetivo caracterizar a cooperação UFSCar-empresa por meio de três programas de inovação tecnológica específicos, enfocando as relações entre os envolvidos no desenvolvimento de projetos de inovação, como mencionado anteriormente.

Para o desenvolvimento do estudo proposto, utilizou-se a pesquisa qualitativa. Essa técnica é a mais adequada quando se objetiva responder questões, por exemplo, de como algum processo e/ou fenômeno social ocorre, como é o caso da pesquisa desenvolvida. Com este estudo buscou-se responder à seguinte questão de pesquisa: como é caracterizada a cooperação UFSCar-empresa por meio dos programas de inovação tecnológica PATME, PITE e PIPE?

Esta pesquisa está inserida em um quadro teórico mais amplo (o da cooperação universidade-empresa) e possui foco de interesse mais específico. Como não há uma massa crítica significativa a respeito do assunto estudado, foi realizada então uma pesquisa exploratória (BECKER, 1993; GODOY, 1995).

Para a realização da pesquisa em questão foi utilizado o método do estudo de caso, um dos instrumentos a serem utilizados em uma pesquisa qualitativa (GODOY, 1995). Esse método foi considerado o mais adequado para ser utilizado nesta investigação, devido à possibilidade de sua utilização poder tornar explícitas informações difíceis de serem identificadas.

A realização desta pesquisa ocorreu em duas etapas. $\mathrm{Na}$ primeira foi realizada uma coleta de dados secundários, ou seja, de dados preexistentes em contratos de projetos de inovação tecnológica, para identificar os envolvidos nos projetos tanto do meio acadêmico como do empresarial. Para determinar os entrevistados foi realizado um levantamento de dados nos contratos registrados na FAI/UFSCar, envolvendo os projetos PATME, e no site da FAPESP, para obter informações a respeito dos envolvidos nos projetos PITE e PIPE. A partir da coleta desses dados, foram realiza- 
das as entrevistas com os envolvidos nos projetos. Posteriormente, em uma outra etapa da pesquisa, foram coletados dados primários por meio de entrevistas realizadas com os responsáveis pelos projetos, identificados a partir da coleta de dados anterior.

Com a realização de entrevistas piloto, foram estabelecidas as questões a serem aplicadas aos entrevistados. Os roteiros das entrevistas foram compostos por questões abertas e semi-estruturadas para possibilitar aos entrevistados a expressão de suas opiniões, sem delimitações prévias, o que não ocorreria se estas questões fossem fechadas e estruturadas.

Algo típico de ocorrer quando se utiliza o método do estudo de caso, é a detecção de variáveis que não haviam sido previstas em um primeiro momento. Analisando os contratos formais que envolvem o processo de cooperação UFSCar-empresa estudado, verificou-se que além da participação formal de alguns pesquisadores da referida universidade há também o envolvimento informal de outros pesquisadores da mesma instituição e da USP/São Carlos nesses projetos. Além disso, durante a realização das entrevistas a pesquisadora foi identificando a participação de outros indivíduos, elevando dessa maneira o número de entrevistados previstos inicialmente. Assim, entre indivíduos do meio acadêmico e do meio empresarial, foram realizadas 45 entrevistas.

As entrevistas foram analisadas, em primeiro lugar, separando os discursos dos entrevistados em pesquisadores, empresários, consultores e alunos, para que estes discursos pudessem ser compreendidos de acordo com suas especificidades. Com isso, pôde-se observar as diferenças e semelhanças entre os discursos e associá-los a categorias específicas.

A partir da análise dos discursos dos entrevistados, foram identificados aspectos como motivações, benefícios, dificuldades, entre outros, associados ao desenvolvimento de projetos de inovação tecnológica envolvendo a UFSCar e o meio empresarial.

Com o interesse em incentivar o aumento do desenvol- vimento de projetos de inovação tecnológica envolvendo universidade e empresa, considera-se relevante destacar os benefícios trazidos por meio da execução desse tipo de projeto.

É importante mencionar que, apesar de a pesquisa qualitativa possuir estratégias de pesquisa diferentes da quantitativa, não se pode considerar que ambas sejam excludentes. Isso quer dizer que é possível realizar, ao mesmo tempo, uma coleta de dados que também possuam características quantitativas.

Desta forma, neste artigo são utilizadas informações qualitativas presentes repetidas vezes nos discursos, que são contadas e tabeladas para facilitar a interpretação e apresentação dos resultados. Na próxima seção são apresentados os benefícios identificados por meio dessa pesquisa.

\section{ANÁLISE DAS ENTREVISTAS}

Como mencionado anteriormente, o objetivo deste artigo é apresentar resultados parciais de um estudo de caso que caracterizou a cooperação entre a UFSCar e o meio empresarial por meio de programas de inovação tecnológica específicos. Esse artigo está focado nos benefícios que os envolvidos em projetos de inovação tecnológica obtiveram com a execução dos mesmos.

No período de dezembro de 2001 a maio de 2002 foram realizadas 45 entrevistas com 16 empresários, 24 pesquisadores, 3 alunos de graduação e 2 consultores do Núcleo de Informação Tecnológica - NIT/Materiais. O número de entrevistas realizadas por programa é apresentado na Tabela 1.

As entrevistas realizadas envolveram 24 pesquisadores, sendo 3 da USP/São Carlos envolvidos em projetos PITE e 21 da UFSCar com projetos PIPE e PATME (2 coordenadores de projetos e 19 pesquisadores que prestaram consultoria a esses e a outros projetos). As demais entrevistas envolveram 2 alunos de graduação da UFSCar e 2 consultores do NIT/Materiais ${ }^{1}$. Com relação aos programas, 23 entrevistas foram realizadas com indivíduos com PIPE, 13 com PATME e 9 com PITE.

Tabela 1: Número de entrevistas realizadas por programa.

\begin{tabular}{|c|c|c|c|c|c|}
\hline $\begin{array}{c}\text { ENTREVISTAS } \\
\text { Programas }\end{array}$ & EMPRESÁRIOS & PESQUISADORES & ALUNOS & CONSULTORES & TOTAL \\
\hline PIPE & 8 & 12 & 3 & 0 & 23 \\
\hline PATME & 5 & 6 & 0 & 2 & 13 \\
\hline PIPE & 3 & 6 & 0 & 0 & 9 \\
\hline Total & 16 & 24 & 3 & 2 & 45 \\
\hline
\end{tabular}

Fonte: Adaptado de Calligaris, 2002. 
As entrevistas foram analisadas, em primeiro lugar, a partir da separação dos discursos dos empresários, pesquisadores, consultores e dos alunos, para que os mesmos pudessem ser compreendidos de acordo com suas particularidades. $\mathrm{O}$ meio em que os entrevistados estavam envolvidos foi considerado importante para a análise da especificidade dos dados.

Como se pretendeu retratar o caso de forma completa, as informações foram coletadas em uma variedade de situações, em diversos momentos e com diferentes fontes de informações (pesquisadores, alunos consultores e empresários). Para isso, utilizou-se a técnica da triangulação (LUDKE \& ANDRÉ, 1986).

Para a análise dos dados coletados, elementos convergentes e divergentes, inerentes aos discursos dos entrevistados, foram associados a diferentes categorias, que foram relacionadas pela pesquisadora a aspectos relativos ao processo de desenvolvimento dos projetos. Esse processo pode ser ilustrado, em uma ordem de acontecimentos envolvendo motivações, dificuldades e benefícios associados à execução desses projetos. Ao analisar as entrevistas, os benefícios foram em agrupados em categorias específicas. A seguir são apresentadas as categorias identificadas neste estudo.

- Inovações: refere-se à obtenção de novos produtos e/ou à realização de melhorias em projetos existentes. Consideram-se os casos com resultados tanto do ponto de vista empresarial quanto acadêmico;

- Obtenção de resultados práticos: considera a possibilidade de ter como retorno imediato um produto no mercado, redução de custos e outros retornos financeiros;

- Pessoais: considera o enriquecimento curricular advindo da participação dos indivíduos nos projetos;

- Financeiros: aborda a obtenção de recursos financeiros concedidos pelos programas, por meio dos quais é possível comprar equipamentos, adquirir bolsas, con- tratar pessoal qualificado, etc;

- Parceria: envolve a troca de informações e experiências entre as partes envolvidas nos projetos;

- Recursos humanos: considera a possibilidade de contratação de pessoal qualificado para o desenvolvimento dos projetos;

- Geração de publicações-patentes: aborda o desenvolvimento de produtos que podem gerar publicação de artigos e participação em congressos; e a geração de royalties em caso de obtenção de patente;

- Outros: envolve o desconhecimento dos benefícios em projetos que estão no início ou durante o processo de desenvolvimento.

Ao analisar os discursos, foram associados os aspectos convergentes a determinadas categorias. As próximas seções apresentam os benefícios identificados às classes de entrevistados.

\section{Benefícios apontados pelos empresários}

Nesta seção são apresentados os benefícios referentes ao desenvolvimento dos projetos apontados pelos empresários, os quais foram associados às categorias apresentadas na Tabela 2. Nessa tabela aparecem as frequiências absolutas dos benefícios.

Na Tabela 2 pode-se observar que as categorias apresentadas com maior freqüências foram: inovações e obtenção de resultados práticos. Os trechos das entrevistas apresentados a seguir ilustram os benefícios diretos que foram associados a essas categorias:

“(...) com o desenvolvimento do projeto fizemos uma melhoria num produto que já existia. Foi um tipo de inovação o que nós fizemos" (empresário com projeto PATME).

Tabela 2: Benefícios apontados pelos empresários.

\begin{tabular}{|c|c|c|c|c|}
\hline Benefícios EMPRESÁRIOS & PATME & PITE & PIPE & TOTAL \\
\hline Inovações & 3 & 1 & 3 & 7 \\
\hline Obtenção de resultados práticos & 0 & 2 & 5 & 7 \\
\hline Financeiros & 1 & 2 & 1 & 4 \\
\hline Recursos humanos & 0 & 0 & 3 & 3 \\
\hline Parceria & 1 & 0 & 1 & 2 \\
\hline Pessoais & 0 & 1 & 0 & 1 \\
\hline Outros & 1 & 0 & 0 & 1 \\
\hline Total & 6 & 6 & 13 & 25 \\
\hline
\end{tabular}

Fonte: Adaptado de Calligaris, 2002. 
“(...) o desenvolvimento de novas formas de controle e monitoramento aplicáveis à produção. Uma inovação que nos trouxe realmente um benefício prático" (empresário com PATME).

“(...) o desenvolvimento de um sistema de impermeabilizantes para coberturas de construções civis" (empresário com PITE). quiridos equipamentos que poderão ser utilizados em projetos futuros" (empresário com projeto PIPE).

A categoria recursos humanos, referente à contratação de pessoal qualificado para o desenvolvimento dos projetos, foi apontada somente por empresários com PIPE, e os benefícios provenientes da categoria pessoais, foram mencionados apenas por empresários com PITE. Os trechos abaixo ilustram os benefícios associados às categorias mencionadas, respectivamente.

“(...) os benefícios foram a capacitação de pessoas da empresa para trabalhar em outros projetos desse e a contratação de recursos humanos da UFSCar para o desenvolvimento do produto" (empresário com PIPE).

“(...) a minha participação e a do coordenador do projeto em palestras, congressos nacionais e internacionais para apresentar o produto desenvolvido foi o grandes benefício do trabalho" (empresário com PITE).

Como no PITE a existência de parceria entre universidade e empresa é requisito do próprio programa, tende-se então a esperar que todos os aspectos referentes a essa categoria ocorram normalmente e, por isso, não são considerados benefícios pelos entrevistados que tiveram projetos PITE. No caso do PATME e do PIPE, apesar da parceria não ser requerida por esses programas diretamente, a ocorrência de troca de informações e experiências entre as partes envolvidas nos projetos foi considerada como benefício por empresários. Alguns benefícios agrupados nessa categoria são ilustrados com os trechos a seguir:

Tabela 3: Benefícios apontados pelos pesquisadores.

\begin{tabular}{|c|c|c|c|c|}
\hline Benefícios PERSQUISADORES & PATME & PITE & PIPE & TOTAL \\
\hline Inovações & 3 & 2 & 3 & 8 \\
\hline Pessoais & 2 & 1 & 3 & 6 \\
\hline Obtenção de resultados práticos & 3 & 1 & 1 & 5 \\
\hline Recursos humanos & 1 & 0 & 4 & 5 \\
\hline Financeiros & 2 & 0 & 2 & 4 \\
\hline Parceria & 1 & 2 & 2 & 5 \\
\hline Geração de publicações-patentes & 0 & 1 & 0 & 1 \\
\hline Total & 12 & 7 & 15 & 34 \\
\hline
\end{tabular}

Fonte: Adaptado de Calligaris, 2002. 
“(...) então os benefícios foram as trocas de informações e as experiências, trabalhar com profissionais gabaritados da universidade e de áreas diferentes como produção, RH, estatística e qualidade" (empresário com projeto PATME).

“(...) a troca de experiências e informações entre o meio acadêmico e empresarial, além da possibilidade da estruturação de uma equipe de trabalho especializada" (empresário com projeto PIPE).

\section{Benefícios apontados pelos pesquisadores}

Nesta seção são apresentadas as freqüências absolutas dos benefícios apontados pelos pesquisadores, de acordo com as categorias a que foram associados, conforme pode ser observado na Tabela 3.

No caso das respostas dos pesquisadores, também houve destaque na freqüência de benefícios associados à categoria inovações, seguidos das freqüências dos benefícios associados às categorias: pessoais, obtenção de resultados práticos, recursos humanos, financeiros, parceria e geração de publicações-patentes.

Como pôde ser observado nessa tabela, os benefícios agrupados na categoria inovações obtiveram destaque. A essa categoria estão associados benefícios tais como os mencionados a seguir:

“(...) tivemos como benefício uma melhoria num produto da empresa, o que foi uma inovação" (pesquisador com projeto PATME).

“(...) a realização de um projeto de cunho tecnológico, o qual seria impossível de ser desenvolvido sem esta parceria" (pesquisador com projeto PITE).

“(...) a construção de um aparelho nacional, com este financiamento, do qual existem similares, mas que são importados" (pesquisador com projeto PIPE).

Os benefícios associados à categoria pessoais podem ser ilustrados por meio dos trechos apresentados a seguir:

“(...) os benefícios foram de aprendizagem científica e tecnológica para mim e para o meu grupo de pesquisa" (pesquisador com projeto PATME).

"(...) o aprendizado individual durante todo o tempo do projeto" (pesquisador com projeto PITE).

"(...) com o desenvolvimento do projeto, houve bene- fícios para o meu dia-a-dia de trabalho devido às experiências que foram realizadas" (pesquisador com projeto PIPE).

Como resultados práticos obtidos com o desenvolvimento dos projetos, os pesquisadores apontaram os seguintes benefícios associados à categoria obtenção de resultados práticos, os quais são ilustrados com os trechos a seguir:

“(...) o desenvolvimento de algo que pôde ser comercializado logo em seguida" (pesquisador com projeto PATME).

“(...) o resultado foi imediato, nós participamos do processo de desenvolvimento desse produto e estamos participando também de sua comercialização" (pesquisador com projeto PITE).

“(...) no final do projeto nós poderemos ver o produto pronto, um produto que será passível de comercialização, e isso é um grande resultado" (pesquisador com projeto PIPE).

Os benefícios associados às categorias recursos humanos e financeiros foram apontados somente por pesquisadores com PATME e PIPE. Para pesquisadores com PAT$\mathrm{ME}$, alguns desses benefícios foram:

“(...) a formação de pessoal, ou seja, de recursos humanos dentro desse espírito, o do programa" (pesquisador com projeto PATME).

“(...) recursos para o laboratório e bolsas para os alunos" (pesquisador com PATME).

\section{desenvolvimento de inovações, que é a
própria finalidade dos programas que financiam os projetos, esteve naturalmente presente nos trechos dos discursos dos entrevistados, tanto do meio acadêmico como do empresarial.}

Para pesquisadores com PIPE, os benefícios associados a estas duas categorias (recursos humanos e financeiros) foram: a formação de recursos humanos e o faturamento que o desenvolvimento do próprio projeto proporciona à empresa. A seguir são apresentados dois trechos para ilustrar os benefícios associados a essas duas categorias, respectivamente:

“(...) através dos contatos obtidos por intermédio da empresa, houve a possibilidade de um aluno que estava participando no projeto fazer um doutorado sanduíche na Espanha" (pesquisador com projeto PIPE).

“(...) o benefício, além do desenvolvimento do projeto, 
foi o faturamento dele decorrente" (pesquisador com PIPE).

A categoria parceria aparece distribuída de forma quase uniforme com relação aos benefícios apontados nos discursos dos pesquisadores com PATME, PIPE e PITE (com frequiências absolutas iguais a 1, 2 e 2, respectivamente). A seguir é apresentado um exemplo para ilustrar esse tipo de benefício.

"(...) o benefício foi a troca de experiências entre as partes e ver que um projeto tem começo, meio e fim" (pesquisador com projeto PIPE).

\section{Síntese dos benefícios identificados e análise}

Esta seção apresenta uma síntese dos resultados, considerando os discursos dos empresários, pesquisadores, consultores e alunos.

Deve-se observar que não houve necessidade de apresentar, separadamente, em tabelas as categorias associadas aos benefícios apontados pelos consultores e entrevistados, devido à baixa frequiência absoluta de suas respostas, o que poderá ser observado na Tabela 4 .

Para uma visualização mais completa dos benefícios apontados pelos entrevistados, foi apresentada essa tabela geral contendo todas as categorias e frequiências absolutas compiladas.

Dentre os benefícios associados às categorias apresentadas na Tabela 4, pode-se observar que a categoria inovações tem a maior frequiência absoluta, igual a 16, dentre as respostas explicitadas pelos entrevistados.

A partir dos dados apresentados neste artigo pôde-se observar que o desenvolvimento de inovações tecnológicas, que é a própria finalidade dos programas que financiam os projetos, esteve naturalmente presente nos trechos dos discursos dos entrevistados, tanto do meio acadêmico como empresarial. Na seqüência aparecem os benefícios associados às categorias: financeiros, recursos humanos e parceria.

Observou-se um destaque para os benefícios associados às categorias pessoais e geração de publicaçõespatentes nos discursos dos pesquisadores. Isso indica que, de certa forma, ao final do desenvolvimento de seus projetos, ocorrem o enriquecimento curricular e a troca de experiências entre as partes envolvidas. Desta maneira, pode-se observar, por meio dos benefícios apontados por esses entrevistados, que os mesmos são motivados a participar dos projetos basicamente pela possibilidade de pesquisa que detectam.

Analisando-se os benefícios identificados nos discursos dos pesquisadores, pode-se perceber que alguns deles, a princípio, não pertencem aos objetivos dos programas de inovação tecnológica da FAPESP. Observou-se que, dentre esses benefícios, parte deles aparecem somente em discursos de pesquisadores com PIPE e com PITE. Por exemplo, questões associadas a recursos humanos, problemas financeiros e de parceria são mencionadas somente nos discursos de pesquisadores com PIPE. Já questões envolvendo o desenvolvimento de interdisciplinares e publicações-patentes aparecem apenas nos discursos de pesquisadores com PITE. 
Como o PITE é um projeto de parceria e que, portanto, envolve contrapartida financeira de empresas, questões relacionadas a problemas financeiros, de parceria e de obtenção de recursos humanos não são preocupações reveladas diretamente por pesquisadores com esse tipo de projeto, mas por pesquisadores com projeto PIPE. Por outro lado, os projetos PIPE são desenvolvidos em pequenas empresas, geralmente com dificuldades financeiras e de recursos humanos para o desenvolvimento de projetos.

Naturalmente, devido a uma maior complexidade, a priori, dos projetos PITE, questões relacionadas ao desenvolvimento de projetos interdisciplinares, à geração de publicações e à busca por patentes são freqüentes nos discursos de pesquisadores com projetos PITE, enquanto que, nos discursos de pesquisadores com PIPE, não são mencionadas.

\section{CONSIDERAC̄ÕES FINAIS}

Este artigo teve como objetivo investigar o funcionamento, o tipo de relacionamento e a eficiência dos programas governamentais de financiamento para projetos que envolvem cooperação. Neste contexto foi realizado um estudo de caso específico envolvendo a UFSCar e o setor empresarial.

Como se trata de uma pesquisa qualitativa, o grau de satisfação dos envolvidos nos projetos quanto à realização dos mesmos é difícil de ser mensurado. Sendo assim, considerou-se a perspectiva dos próprios entrevistados com relação a sua satisfação ou não quanto aos resultados obtidos. Pode-se observar que, de um modo geral, esses entrevistados estão satisfeitos com os resultados de seus projetos, o que pode ser constatado por meio dos benefícios que os mesmos apontaram em seus discursos.

\section{e orientar o desenvolvimento de outros projetos de inovação tecnológica.}

Os benefícios identificados nesta pesquisa constituem variáveis implícitas que foram explicitadas por meio de pesquisa qualitativa. Os benefícios identificados são importantes no sentido de incentivar e orientar o desenvolvimento de outros projetos de inovação tecnológica com interação universidade-empresa financiados por programas governamentais.

Os modelos teóricos mencionados, que abordam a cooperação universidade-empresa-governo, não discutem aspectos referentes, por exemplo, ao processo de desenvolvimento de projetos de inovação tecnológica tais como os benefícios oriundos desse processo e as dificuldades em

Tabela 4: Freqüências absolutas dos benefícios apontados pelos entrevistados.

\begin{tabular}{|c|c|c|c|c|c|c|c|c|c|}
\hline \multirow{2}{*}{$\begin{array}{c}\text { ENTREVISTADOS } \\
\text { Benefícios }\end{array}$} & \multicolumn{3}{|c|}{ EMPRESÁRIOS } & \multicolumn{3}{|c|}{ PESQUISADORES } & \multirow{2}{*}{$\begin{array}{c}\text { CONSULTORES } \\
\text { PATME }\end{array}$} & \multirow{2}{*}{$\frac{\text { BOLSISTAS }}{\text { PIPE }}$} & \multirow[t]{2}{*}{ TOTAL } \\
\hline & PATME & PITE & PIPE & PATME & PITE & PIPE & & & \\
\hline Inovações & 3 & 1 & 3 & 3 & 2 & 3 & 1 & 0 & 16 \\
\hline $\begin{array}{l}\text { Obtenção de } \\
\text { resultados práticos }\end{array}$ & 0 & 2 & 5 & 3 & 1 & 1 & 2 & 0 & 14 \\
\hline Financeiros & 1 & 2 & 1 & 2 & 0 & 2 & 0 & 0 & 8 \\
\hline Recursos humanos & 0 & 0 & 3 & 1 & 0 & 4 & 0 & 0 & 8 \\
\hline Parceria & 1 & 0 & 1 & 1 & 2 & 2 & 0 & 0 & 7 \\
\hline Pessoais & 0 & 1 & 0 & 2 & 1 & 3 & 0 & 0 & 7 \\
\hline $\begin{array}{l}\text { Geração } \\
\text { publicações-patentes }\end{array}$ & 0 & 0 & 0 & 0 & 1 & 0 & 0 & 0 & 1 \\
\hline Outros & 1 & 0 & 0 & 0 & 0 & 0 & 0 & 3 & 4 \\
\hline Total & 6 & 6 & 13 & 12 & 7 & 15 & 3 & 3 & 65 \\
\hline
\end{tabular}

Fonte: Adaptado de Calligaris, 2002. 
obtê-los conforme apresentado neste artigo.

Nesse sentido, a continuidade das investigações desse tipo de relação entre universidade, empresa e governo pode permitir um maior detalhamento das mesmas. Um melhor conhecimento dessas relações pode facilitar a elaboração até mesmo de estratégias eficientes para a otimização de projetos de inovação tecnológica, incentivando o aumento do número de participantes em projetos desse tipo, bem como um melhor aproveitamento dos investimentos em tecnologia feitos pelo governo e a iniciativa privada no País.

\section{Edição especial início 07/03/2003 fim 25/08/2003}

\section{- Referências Bibliográficas}

BECKER, H. A. Métodos de pesquisa em Ciências Sociais. São Paulo, Hucitec, 1993.

BRISOLLA, S. N., et al. Relatório Final: o papel da universidade na pesquisa aplicada e a formação de profissionais - um estudo de caso da Unicamp. Brisolla (coord.). Campinas: Abril, 1998. 143p.

CALLIGARIS, A. B. A ccoperação UFSCar-empresa por meio dos programas de inovação tecnológica PATME, PITE e PIPE. Dissertação (Mestrado). Departamento de Engenharia de Produção Universidade Federal de São Carlos. São Carlos, set. 2002, 200 p.

CRUZ, C.H.B. Universidade, Empresa e a Inovação Tecnológica. In: PLONSKI A.G. (coord.). Interação Universidade-Empresa. Brasília: Instituto Brasileiro de Informação em Ciência e Tecnologia (IBICT), 1998, v.1, p. 226-240.

CRUZ, C.H.B. A universidade, a empresa e a pesquisa que o país precisa. Revisto Parcerias Estratégicas. n. 8, p. 5-30, Brasília, maio, 2000.
ETZKOWITZ, H.; LEYDESDORFF, L. The dynamics of innovation: from National Systems and "Mode 2" to a Triple Helix of university-industry-government relations. Research Policy, v. 29 , Issue 2 , p.109-123, 2000.

FERRO, J.R.(a) Administração da Tecnologia na Universidade e na Empresa. In: FERRO, J.R. (coord.). Universidade e Indústria: depoimentos. $2^{\circ}$ ed. São Carlos : Ed. da UFSCar, p.920, 1997.

GODOY, A.S. Pesquisa Qualitativa Tipos fundamentais. Revista de Administração.

LEYDESDORFF, L.; ETZKOWITZ, H. The Triple Helix - University-IndustryGovernment Relations: A Laboratory for Knowledge Based Economic Development, 1995. Theme paper, WORKSHOP, Amsterdam, 4-6 January, 1996

LUDKE, M.; ANDRÉ, M. E. D. A. Métodos de coleta de dados: observação, entrevista e análise documental. In: Pesquisa e educação: abordagens qualitativas. São Paulo. E.P.U, p. 25-45, 1986.
NELSON, R.R.; ROSEMBERG, G.N Technical Innovation and Nacional Systems. In: NELSON, R. R., (ed.) National Innovation Systems - A Comparative Analysis. New York : Oxford University Press, 1993.

OLIVEIRA, R.M. A cooperação da UFSCa com o meio externo. Dissertação (Mestrado). Departamento de Engenharia de Produção - Universidade Federa de São Carlos. São Carlos, Set. 2001 $134 \mathrm{p}$.

PLONSKI, G. A. Cooperação empresauniversidade no brasil: um novo balanç prospectivo. In: PLONSKI A.G. (coord.) Interação universidade-empresa. Brasília Instituto Brasileiro de Informação em Ciência e Tecnologia (IBICT), 1998, v. 1, p. 09-23.

PORTER, M.E. A vantagem competitiva das nações. Rio de Janeiro : Campus, 1993.

PREDRASSOLI, J.; SILVA, J.C.T. da FERREIRA, D. Análise de aspectos tecnológicos das empresas e sua relação com o meio ambiente. VIII Simpósio de Engenharia de Produção (SIMPEP), Anais ... Bauru : SP, 12 a 14 de novembro de 2001.
SÁBATO, J. Sobre la autonomia tecnológica. In: GOMES, S.F.\& LEITE, R. C. C. (editores). Ciência, tecnologia e independência. São Paulo : Livraria Duas Cidades, 1978. P. 59-74.

SCHUMPETER, J. A Teoria do Desenvolvimento Econômico. São Paulo : Editora Abril, 1982.

SEGATTO, A.P.; SBRAGIA, R Motivadores, barreiras e instrumentos da cooperação universidade-empresa para as universidades. In: Simpósio da Gestão da Inovação Tecnológica, 19. Anais... São Paulo, 1996.

SILVA, J.C.T Modelo interativo empresauniversidade no desenvolvimento de produto. São Paulo.163 p. Tese (Doutorado) - Escola Politécnica da Universidade de São Paulo. Departamento de Engenharia de Produção, 1999.

SUTZ, J. The university-industrygovernment relations in Latin America. Research Policy. Amsterdam, v.29, p.279290, Feb., 2000

\section{Agradecimentos}

À Fundação de Amparo à Pesquisa do Estado de São Paulo (FAPESP) pelo financiamento da pesquisa e aos entrevistados pela disponibilidade para as entrevistas.

${ }^{1}$ O Núcleo de Informação Tecnológica da Engenharia de Materiais da UFSCar desenvolve projetos com o meio empresarial, possuindo consultores específicos para esta finalidade. 